\title{
COUPLED FINITE ELEMENT-MONTE CARLO SIMULATION OF MICROSTRUCTURE AND TEXTURE EVOLUTION DURING THERMOMECHANICAL PROCESSING
}

\author{
B. Radhakrishnan, G. Sarma and T. Zacharia \\ Oak Ridge National Laboratory \\ P.O. Box 2008, MS-6140, Oak Ridge, TN 37831-6140, USA
}

RECEIVED

JUN 241998

OSTI

\begin{abstract}
A novel simulation technique for predicting the microstructure and texture evolution during thermomechanical processing is presented. The technique involves coupling a finite element microstructural deformation model based on crystal plasticity with a Monte Carlo simulation of recovery and recrystallization. The finite element model captures the stored energy and the crystallographic orientation distributions in the deformed microstructure. The Monte Carlo simulation captures the microstructural evolution associated with recovery and recrystallization. A unique feature of the Monte Carlo simulation is that it treats recrystallization as a heterogeneous subgrain growth process, thus providing the natural link between nucleation and growth phenomena, and quantifying the role of recovery in these phenomena. Different nucleation mechanisms based on heterogeneous subgrain growth as well as strain induced boundary migration are automatically included in the recrystallization simulation. The simulations are shown to account for the extent of prior deformation on the microstructure and kinetics of recrystallization during subsequent annealing. The simulations also capture the influence of the presence of cube orientations in the initial microstructure, and the operation of non-octahedral slip during deformation of fcc polycrystals, on the recrystallization texture.
\end{abstract}

\footnotetext{
The submitted manuscript has been authored by a contractor of the U.S. Government under contract No. DE-AC05-96OR22464. Accordingly, the U.S. Government retains a non-exclusive, royaltyU.S. Government retains a non-exclusive, royalty-
free license to publish or reproduce the published free license to publish or reproduce the published
form of this contribution, or allow others to do so, for U.S. Government purposes.
}

\section{MASTER}

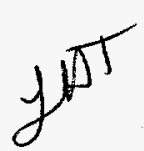

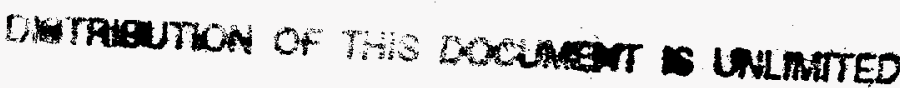




\section{DISCLAIMER}

This report was prepared as an account of work sponsored by an agency of the United States Government. Neither the United States Government nor any agency thereof, nor any of their employees, makes any warranty, express or implied, or assumes any legal liability or responsibility for the accuracy, completeness, or usefulness of any information, apparatus, product, or process disclosed, or represents that its use would not infringe privately owned rights. Reference herein to any specific commercial product, process, or service by trade name, trademark, manufacturer, or otherwise does not necessarily constitute or imply its endorsement, recommendation, or favoring by the United States Government or any agency thereof. The views and opinions of authors expressed herein do not necessarily state or reflect those of the United States Government or any agency thereof. 


\section{DISCLAIMER}

Portions of this document may be illegible in electronic image products. Images are produced from the best available original document. 


\section{Introduction}

In the last decade, several models based on the Monte Carlo (MC) technique have been developed for simulating the temporal evolution of recrystallization microstructures, as well as for predicting the recrystallization kinetics [1-4]. The MC technique involves representing the continuum microstructure on a discrete set of regularly spaced grid of points ( $M C$ grid), associating a volume of material with each point, and evolving the microstructure according to some rules [1]. Each site is assigned a number which represents a crystallographic orientation, so that a grain is defined by a collection of grid points with the same number. Grain boundaries are not represented physically, but are assumed to exist between points with different orientation numbers. The nucleation process is not modeled explicitly. Rather, nuclei are added arbitrarily to the microstructure either in full at the start of the simulation to capture site saturation effects, or continuously as a function of simulation time to model constant nucleation rate effects. The recrystallization model proceeds by randomly selecting grid points and changing the orientation number of the selected point to that of one of its nearest neighbors based on energy considerations. The energy of the point and its neighbors is computed before and after the change, with the change being allowed only if it leads to a reduction in energy. Such a reduction in energy occurs when the orientation of a cold-worked site is converted to that of a recrystallized neighbor, thus causing the movement of the boundaries between cold-worked and recrystallized regions.

In the above simulations [1], the stored energy of deformation was assumed to be uniform and the nuclei were placed randomly in the microstructure. The simulations produced the theoretically expected values of the Johnson-Mehl-Avrami-Kolmogorov [JMAK] exponents for site-saturated and constant nucleation rate conditions. Subsequently, these simulations were extended to the case of heterogeneous nucleation by imposing certain conditions on the relative magnitudes of the stored energy of deformation and the grain boundary energy [2]. The deviation from ideal JMAK behavior displayed by real materials due to non-uniform stored energy was introduced in $\mathrm{MC}$ simulations [4] by varying the stored energy of a site as a function of its orientation number, thus resulting in a range of stored energy values for the cold-worked sites. The simulations showed significant deviations from ideal JMAK kinetics, as is commonly observed in real materials.

Recently, the MC simulation of recrystallization was coupled to a finite element (FE) simulation of microstructural deformation based on crystal plasticity $[5,6]$. The crystal plasticity model provided a quantitative description of the stored energy and orientation distributions in the deformed microstructure. This allowed the rigorous representation of the crystallographic orientations in the deformed microstructure in the form of axis-angle pairs rather than by orientation numbers. The crystal plasticity model also provided a quantitative description of the stored energy of deformation at each $\mathrm{MC}$ site. The dislocation substructure at each MC site was assumed to consist of a collection of subgrains, whose mean diameter and misorientation were calculated from the stored energy and plastic strain at each site. A nucleation model was built into the recrystallization simulation based on analytical modeling of subgrain growth kinetics. The stored energy of each site was reduced with simulation time based on subgrain growth kinetics, and therefore simultaneous recovery during recrystallization was also included in the simulation. The coupled FE-MC model was able to provide, for the first time, quantitative information on the evolution of microtexture during recrystallization. The model also quantified the role of recovery during recrystallization. 
In all of the above models, recrystallization is represented as a two-step process involving distinct nucleation and growth stages, similar to the classic diffusional phase transformations. This is because of the general observation that recrystallization is characterized by the presence of an incubation phase during which many locations in the deformed microstructure "nucleate" strain-free grains generally bounded by high-angle boundaries, followed by a growth phase in which these boundaries sweep through the deformed microstructure. However, it is known that the mechanism of nucleation during recrystallization is different from that in phase transformations in that the nuclei either already exist in the deformed microstructure in the form of supercritical subgrains/cells or they form by the heterogeneous growth of pre-existing subcells/grains $[7,8]$ at certain locations. The growth phase simply involves the continued heterogeneous movement of the nuclei boundaries into the not yet evolved regions of the deformed microstructure.

In this paper, a new model for recrystallization is presented where recrystallization is modeled as a heterogeneous subgrain growth process. The quantitative description of the deformed microstructure provided by the FE model is mapped to a regular grid of points, each of which is characterized by a stored energy, a plastic strain and a crystallographic orientation. The substructure at each site in the form of a subgrain network is then obtained from a knowledge of the plastic strain and the stored energy at the site, and a subgrain structure is assembled for the whole simulation domain. The growth of the heterogeneous subgrain network is then simulated by using a Monte Carlo technique. The simulations are shown to capture the formation of nuclei by heterogeneous subgrain growth. The simulations also indicate how existing prior high angle boundaries as well as the high angle boundaries produced by the deformation process migrate to sweep through the remaining deformed regions to produce typical recrystallized microstructures and kinetics.

The above simulation technique is used to evaluate the recrystallization kinetics, microstructure and texture evolution for deformed fcc polycrystals. A major focus of the simulations is to evaluate the influence of the operation of non-octahedral slip systems during hot deformation $[9,10]$ on the recrystallization texture, for initial microstructures with and without cube texture. The simulations show that in the presence of cube texture in the initial microstructure, cube nuclei form both in the bulk of the deformed cube grains and at the boundary between the cube grain and the adjacent grain of non-cube orientation. The growth of the cube nuclei to consume other non-cube orientations during recrystallization depends on the local substructure produced by deformation. In the absence of initial cube orientations in the microstructure, the deformation texture does not contain cube orientations for the deformations of $50 \%$ and $68 \%$ used in this study. However, subsequent annealing tends to produce orientations close to cube in certain regions of the microstructure, and a rotated cube orientation in certain other locations.

\section{Computational Approach}

The computational approach used in this study involves coupling a FE microstructural deformation model based on crystal plasticity with a Monte Carlo simulation of recrystallization. The FE model is described in a companion paper in these proceedings [11]. The initial microstructure is discretized such that there are several hundred elements per grain. The unique feature of the FE simulations is that it captures the heterogeneity of deformation at the scale of the microstructure and provides a quantitative description of the stored energy, plastic strain and crystallographic orientation in the deformed microstructure. 
In order to utilize the above microstructural information in the recrystallization simulation, it has to be mapped from a deformed $\mathrm{FE}$ mesh to a regular mesh used in the $\mathrm{MC}$ simulations. The mapping is carried out by using a procedure described elsewhere [6]. The deformation substructure at each grid point is then obtained from a knowledge of the stored energy per unit volume at the site. Each site is assumed to consist of a subgrain network with a mean average subgrain size. The stored energy per unit volume at a site, $H$, is assumed to be equal to the energy per unit volume of the subgrain network given by [12],

$$
H=\frac{2 \gamma}{D}
$$

where $D$ is the mean subgrain size and $\gamma$ is the energy per unit area of a subgrain boundary given by

$$
\gamma= \begin{cases}\gamma_{m} \frac{\theta}{\theta^{*}}\left[1-\ln \left(\frac{\theta}{\theta^{*}}\right)\right] & \text { when } \theta \leq \theta^{*} \\ \gamma_{m} & \text { when } \theta>\theta^{*}\end{cases}
$$

where $\theta$ is the misorientation between two subgrains, $\gamma_{m}$ is energy per unit area of a high-angle boundary, and $\theta^{*}$ is the misorientation limit for low angle boundaries, which is usually taken as $15^{\circ}$.

The above equations are used to calculate the mean misorientation between subgrains at every site, assuming that the mean subgrain size is the same for all sites. The calculations result in a local sublattice for each site which contains a subgrain network with a known misorientation between the subgrains. These sublattices are assembled to generate a global lattice which describes the deformation substructure for the entire volume.

The recrystallization simulation is carried out as a subgrain growth phenomenon using a Monte Carlo technique. In the MC simulations, each site is visited in a random fashion and the local energy of the site and its neighborhood, $E_{\text {init }}$, is calculated using,

$$
E_{\text {init }}=\sum_{i} \gamma_{i}
$$

where $\gamma_{i}$ is the specific boundary energy between the site and its $i^{\text {th }}$ neighbor. $\gamma_{i}$ is a function of the misorientation between the two sites, as in equation (2). The misorientation, $\theta$, is calculated using the axis-angle pairs for the two sites.

The energy of the site and its neighborhood when the site is replaced by one of its nearest neighbors, $E_{f i n}$ is then calculated as,

$$
E_{f i n}=\sum_{i} \gamma_{i}^{\prime}
$$

where $\gamma_{i}^{\prime}$ is the orientation dependent specific boundary energy between the replaced neighboring orientation and the original neighborhood of the site. The local energy change $\Delta E$ is calculated as $E_{f i n}-E_{\text {init }}$. The probability of flipping the site to the orientation of the chosen nearest neighbor, $p$, is calculated as,

$$
p=\left\{\begin{array}{cc}
k^{\prime} & \Delta E \leq 0 \\
k^{\prime} \exp (-\Delta E / k T) & \Delta E>0
\end{array}\right.
$$

where $k$ is Boltzmann's constant and $k^{\prime}$ is the misorientation-dependent boundary mobility given by [13]

$$
k^{\prime}=\left[1-\exp \left(-q \theta^{3}\right)\right] \text {, }
$$


where $q$ is a constant, assumed to be 0.001 in the current simulations. In equation (5), the quantity $k T$ is not the absolute value of the thermal energy. It is a relative thermal energy which is typically expressed as a fraction of the thermal energy at the critical temperature (temperature at which the system is fully disordered) in MC simulations. In the current simulations, the quantity $k T$ is assumed to be equal to 0.4 . The reorientation of the site is implemented with the probability $p$ calculated using equation (5) and equation (6).

\section{Simulations}

The computational approach described above was used to simulate the recrystallization kinetics, microstructure and texture evolution for various deformation conditions described below:

1. microstructure without initial cube orientation deformed $50 \%$ using 12 octahedral $\{111\}\langle 110\rangle$ slip systems,

2. microstructure without initial cube orientation deformed $50 \%$ using 18 slip systems consisting of 12 octahedral $\{111\}\langle 110\rangle$ slip systems and 6 non-octahedral $\{110\}\langle 110\rangle$ slip systems,

3. microstructure containing a cube oriented grain deformed $50 \%$ with 12 octahedral slip systems,

4. microstructure containing a cube oriented grain deformed $50 \%$ with 18 slip systems, and

5. microstructure without cube orientation deformed to $68 \%$ using 12 octahedral slip systems.

The deformed microstructure in each case was first mapped to a $30 \times 30 \times 30$ grid. In order to obtain a statistical representation of the subgrain network for each site, a reasonable sublattice size for each site is $15 \times 15 \times 15$. The overall size of the MC mesh in this case becomes $450 \times 450 \times 450$. However, this problem size is too large for single processor workstations. Hence, it was decided to carry out the recrystallization simulations in two-dimensions by taking a section of the $30 \times 30 \times 30$ lattice and using a sublattice size of $15 \times 15$ for each site. In the current simulations the midsection containing the rolling and normal directions is used. The resulting MC grid size of 450 $\times 450$ is easily handled by single processor workstations. However, work is underway to implement the recrystallization simulation on a parallel computing platform, so that a full three-dimensional simulation can be carried out. The recrystallization kinetics, temporal evolution of the recrystallized microstructure and texture are obtained for each of the deformation microstructures listed above.

\section{Results and Discussion}

Fig. 1(a) shows the expected positions of prior grain boundaries in the deformed microstructure for case 3 , assuming that the deformation occurs uniformly throughout the microstructure. Fig. 1(b) shows the deformed microstructure for the same section obtained by the FE simulation, showing the locations of boundaries with misorientations greater than or equal to $15^{\circ}$. It is clear that the inhomogeneous deformation at the scale of the microstructure has resulted in the formation of several additional high angle boundaries. The grain located at the top left hand corner of Fig. 1(b) had cube orientation in the initial microstructure. The heterogeneous deformation has resulted in the formation 


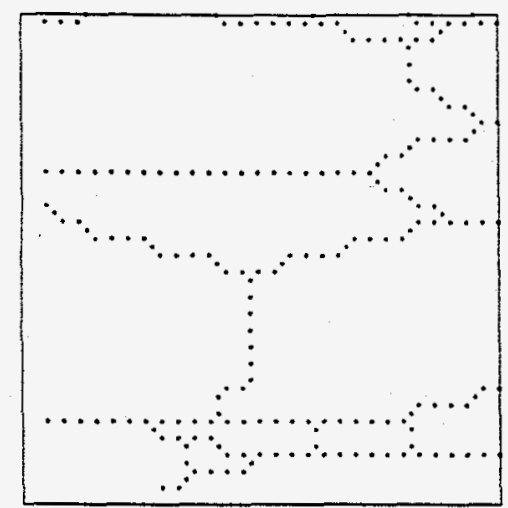

(a)

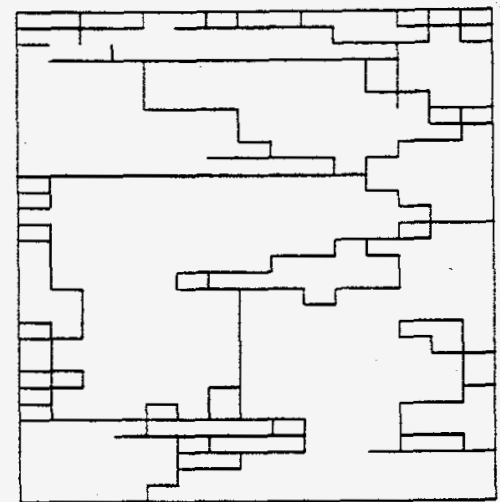

(b)

Figure 1: Deformed grain structures after $50 \%$ deformation with 12 slip systems for initial microstructure with cube grain (a) assuming uniform deformation and (b) FE results.

of a high angle boundary which runs roughly at $45^{\circ}$, probably indicating the formation of a shear band. Extensive investigations of deformation substructures by transmission electron microscopy [14] have revealed that such strain localization and local variations in strain path do result in the formation of deformation induced high angle boundaries. The high angle boundaries formed by the deformation process, as well as some segments of the initial high angle boundaries are potential sites for nucleation.

The temporal evolution of the recrystallization process is shown in Fig. 2 for the deformed grain structure of Fig. 1(b). The high angle and low angle boundaries (misorientation $<15^{\circ}$ ) are indicated by dark and light lines, respectively. The discontinuous subgrain growth originating at the high angle boundaries results in the sweeping of the deformed microstructure by the migrating high angle boundaries, thus simulating recrystallization. In regions where high angle boundaries do not exist, discontinuous growth of subgrains occurs when certain subgrains gain a growth advantage over the neighboring subgrains, probably induced by a long range orientation gradient. Such a discontinuous growth can ultimately lead to the formation of a high angle boundary. However, it is important to note that the recrystallized microstructure contains both high angle and low angle boundaries. The unique feature of the recrystallization simulation is that it is not necessary to invoke a separate nucleation model as was done in a previous study [6]. All the potential nucleation mechanisms operate automatically depending upon the local substructure. Fig. 3 shows the recrystallized microstructure after a Monte Carlo Step (MCS) of 500 for deformation conditions 1 and 5 listed above. Note that higher prior deformation leads to a finer recrystallized grain size. In Fig. 3(a), the recrystallization is not complete since regions with a fine substructure are still present. However, such regions are almost absent in Fig. 3(b) indicating complete recrystallization. This is due to the more uniform distribution of deformation induced high angle boundaries in the latter case. In both cases, discontinuous subgrain growth has resulted in a wide grain size distribution.

The recrystallization kinetics for the various deformation conditions are shown in Fig. 4(a). The variation of recrystallized volume fraction with time shows the typically observed sigmoidal kinetics. However, significant deviation from typical JMAK kinetics is obtained as shown in Fig. 4(b). Prior deformation of $68 \%$ has clearly resulted in a significant acceleration of the recrystallization kinetics compared to the kinetics for a prior deformation of $50 \%$. This is due to the higher stored energy in the former case which results 


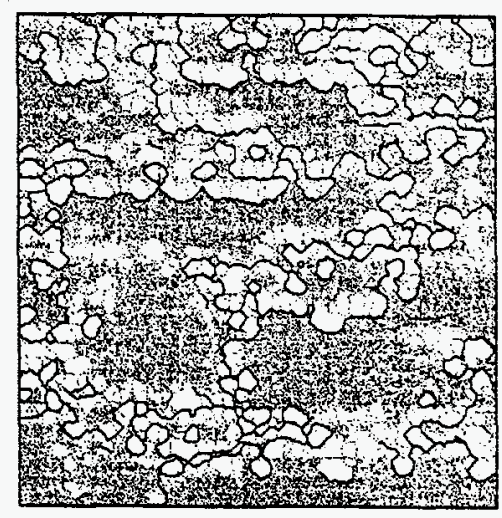

MCS $=100$

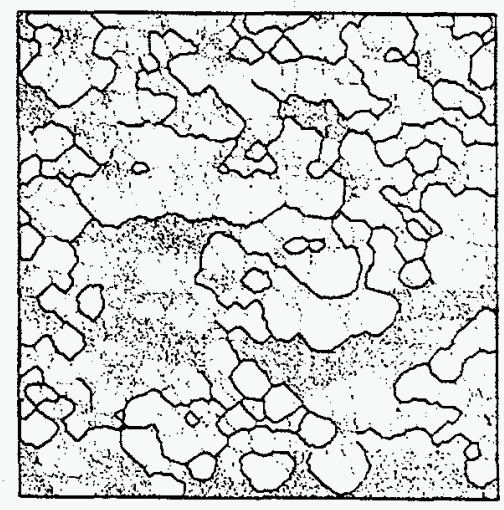

$\mathrm{MCS}=300$

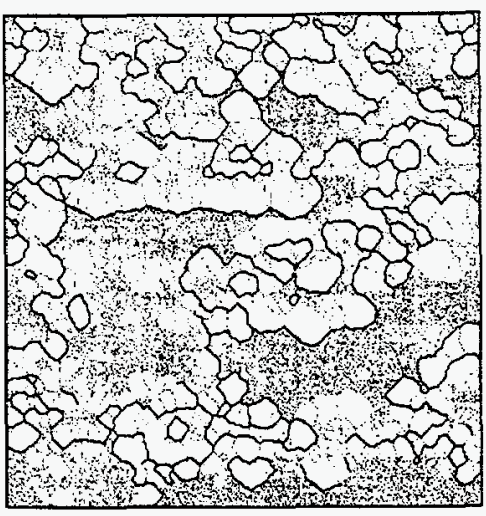

$\mathrm{MCS}=200$

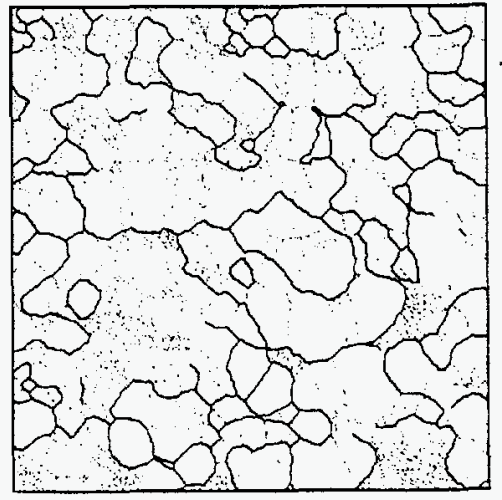

$\mathrm{MCS}=500$

Figure 2: Temporal evolution of recrystallized microstructure for the deformed microstructure shown in Fig. 1.

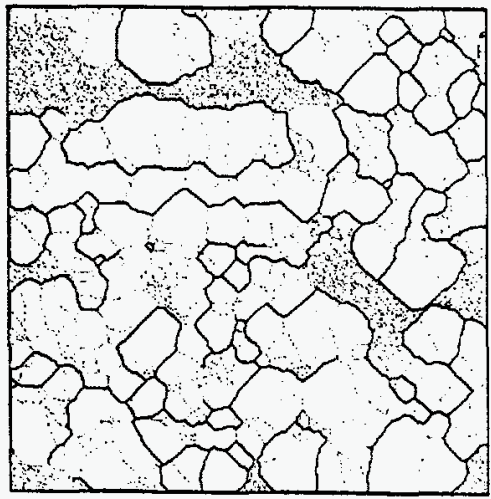

(a) $50 \%$ deformation

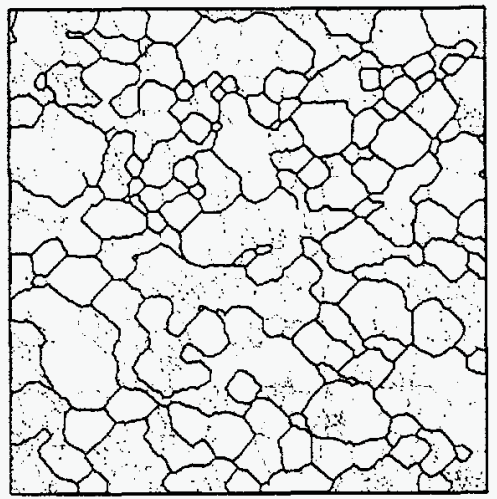

(b) $68 \%$ deformation

Figure 3: Recrystallized microstructure after MCS $=500$ for microstructures without initial cube grain deformed with 12 slip systems. 

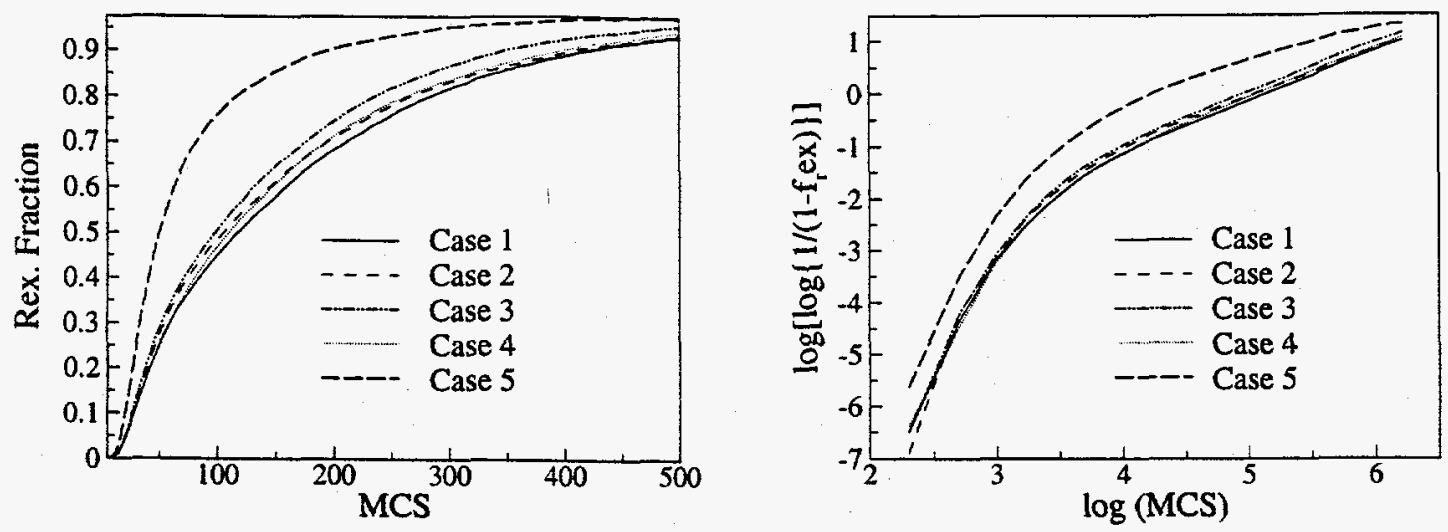

Figure 4: Recrystallized kinetics for the deformation conditions used in this study.

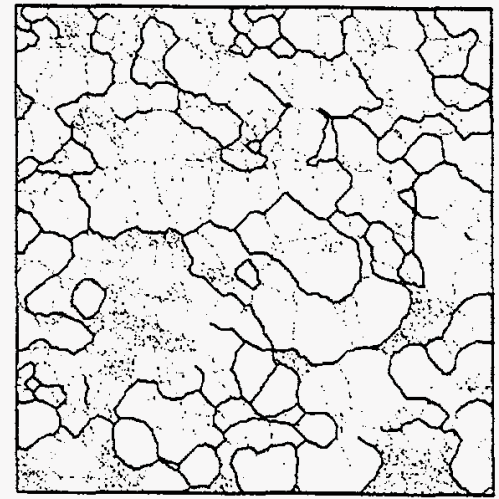

(a) 12 slip systems

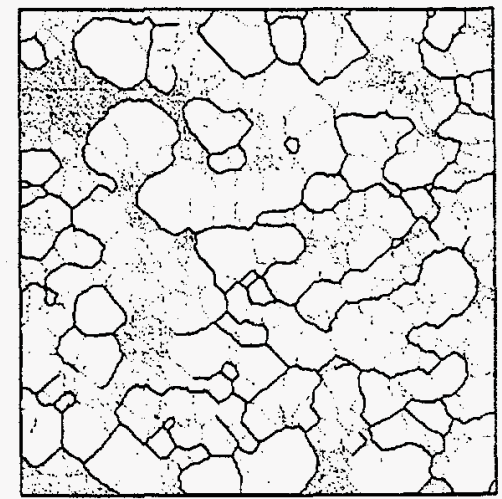

(b) 18 slip systems

Figure 5: Recrystallized microstructures after $\mathrm{MCS}=500$ for initial microstructure containing cube grain and deformed with (a) 12 slip systems and (b) 18 slip systems.

in greater misorientation between subgrains in the deformed microstructure, leading to a greater mobility of the subgrain boundaries. Minor differences in recrystallization kinetics are observed for the deformation conditions 1 through 4 described previously, due to variations in the evolution of subgrain structure as described below.

For the same cube containing initial microstructure, deformation with 12 slip systems (case 3 ) seems to result in faster recrystallization kinetics than deformation with 18 slip systems (case 4). This may be attributed to the noticeable difference in the recrystallization of the initial cube grain between the two cases as shown in Fig. 5. The cube grain in the upper left hand corner of Figs. 5(a) and (b) is seen to evolve differently. For the 12 slip system deformation case shown in Fig. 5(a), the heterogeneous growth of subgrains inside the deformed cube grains appears to be faster than in Fig. 5(b) probably because of greater orientation gradients. Therefore, there are more recrystallization fronts within the cube grain in for the 12 slip system case.

The recrystallization kinetics seems to improve when the initial microstructure prior to deformation contains a cube orientation. This is seen by comparing the microstructures shown in Fig. 3(a) and Fig. 5(a). The formation of high angle boundaries inside the cube grain after deformation as shown in Fig. 1(b) caused a more complete recrystallization 

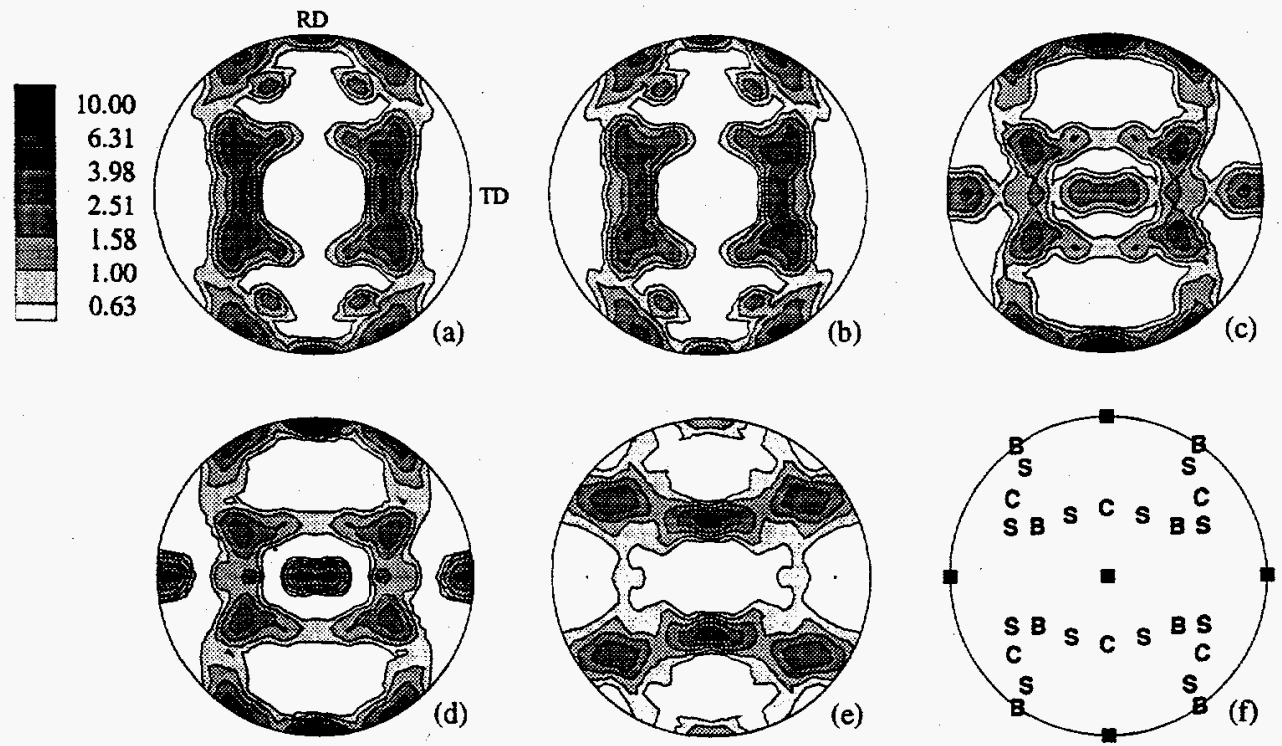

Figure 6: $\langle 100\rangle$ pole figures of the deformation textures for the deformation conditions used in this study. (a)-(e) represent deformation conditions $1-5$ listed in the text. (f) shows some ideal orientations (B: brass, C: copper, S: S, square: cube).

of the cube grain as shown in Fig. 5(a). However, since such a high angle boundary did not form for deformation condition 1, recrystallization of the corresponding grain in Fig. 3(a) had to be initiated by the formation of a nucleus by subgrain growth in orientation gradient, which is a slower process.

Fig. 6 shows the $\langle 100\rangle$ pole figures of the corresponding sections for the deformation conditions used in this study. Fig. $6(\mathrm{f})$ shows the locations of $\langle 100\rangle$ poles for some of the typical orientations found in the deformation textures of fec polycrystals. For microstructures which do not contain initial cube orientation, deformations with both 12 and 18 slip systems produce the commonly observed $\mathrm{S}$, copper and brass textures, as shown in Figs. 6(a), (b) and (e). The deformation textures contain cube orientations only when they are also present in the initial microstructure. For microstructures which contain initial cube orientation, deformation with 18 slip systems results in a stronger remnant cube texture than deformation with 12 slip systems, as seen by comparing Figs. 6(c) and (d) (see also [11]).

Fig. 7 shows the $\langle 100\rangle$ pole figures of the recrystallization textures corresponding to the deformation textures shown in Fig. 6. For microstructures which do not have initial cube orientations, there is a general redistribution of the intensities of the $\mathrm{S}$, copper and brass components after recrystallization, with no indication of cube texture formation. However, for the $68 \%$ deformation with 12 slip systems, there is a tendency for the formation of a rotated cube characterized by a $23^{\circ}$ rotation about the rolling direction, as seen by comparing Fig. 6(e) and Fig. 7(e). These orientations appear along the TD in Fig. 7(e). When the microstructure containing initial cube is deformed using 12 slip systems, the intensity of cube orientation after recrystallization shows a significant increase as seen by comparing Fig. 6(c) and Fig. 7(c). However, when deformed with 18 slip systems, there is no apparent change in texture after recrystallization, as seen by comparing Fig. 6(d) and Fig. 7 (d). The reason for the above difference in recrystallization textures can be obtained from Fig. 5(a) and Fig. 5(b). For the 12 slip system deformation case shown in Fig. 5(a), the recrystallization front from the cube grain is seen to extend downward to consume the 

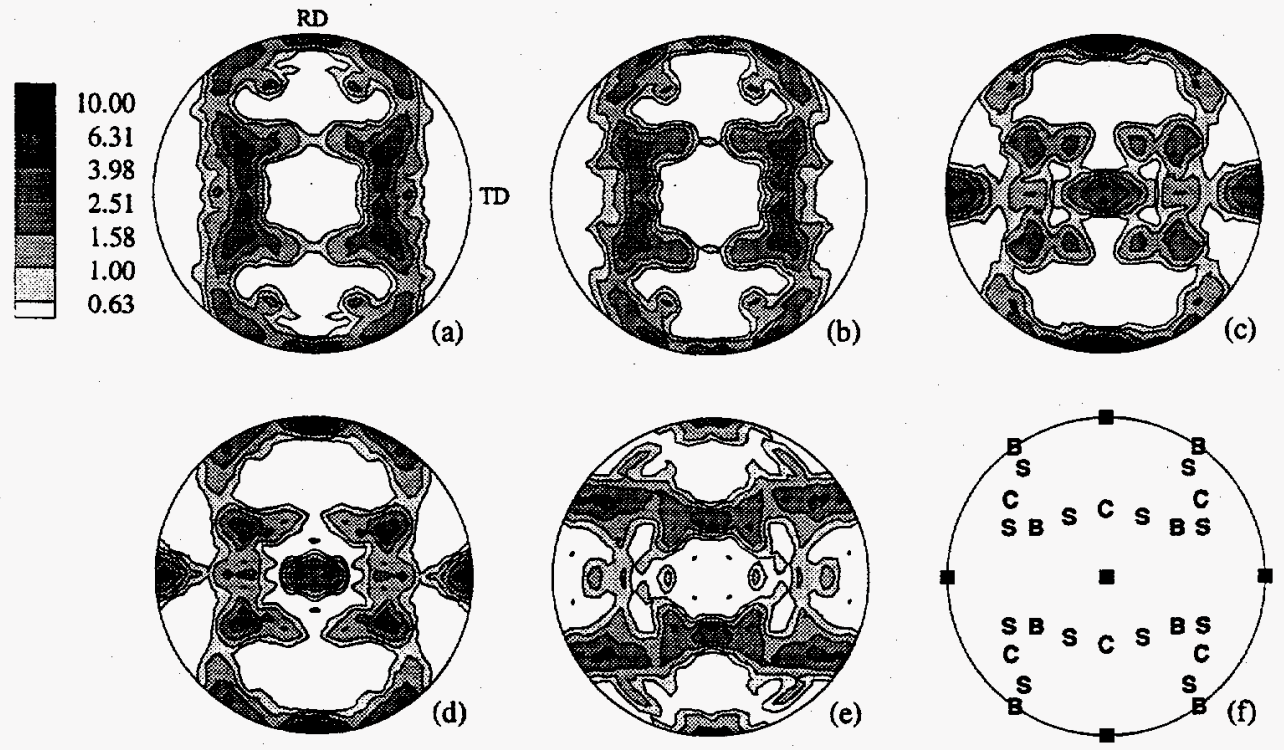

Figure 7: $\langle 100\rangle$ pole figures of recrystallization textures corresponding to the deformation textures shown in Fig. 6.
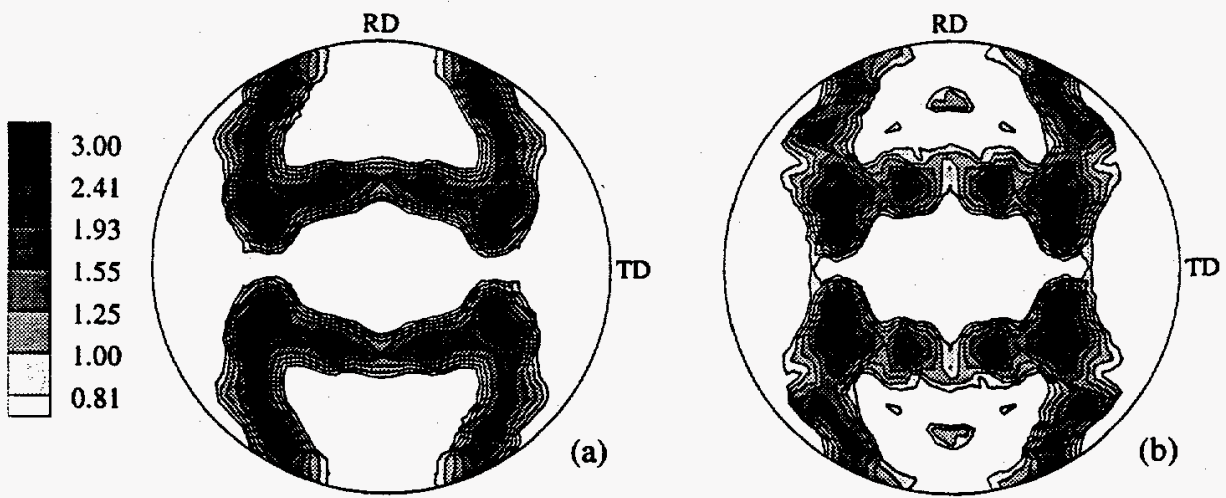

Figure 8: Deformation and recrystallization textures for the deformation condition 1 from a different section of the deformation volume than in Figs. 6 and 7.

grain below, thus increasing the cube intensity (see Fig. 1(b) for the initial positions of the high angle boundaries and Fig. 2 for their evolution). However, for the 18 slip system deformation case shown in Fig. 5(b), the recrystallization fronts at the boundary of the cube grain and the grain below it tend to move in opposite directions, with one front increasing the cube intensity and the other decreasing it. The outward migration of the recrystallization front from the boundary of the cube grain to consume other deformation structures is similar to the mechanism of cube texture formation suggested by Vatne et al. [15].

Fig 8 shows the deformation and recrystallization textures for a different section than the one in Fig. 6(a) and Fig. 7(a) of the deformation volume deformed with 12 slip systems. Fig 9 shows the corresponding textures for the same section deformed with 18 slip systems. The initial orientations in this section were such that a small amount of near-cube orientations were produced by deformation with 18 slip systems but not by deformation with 12 slip systems. However, the volume fraction of this component is so small that there are no major differences in the deformation textures shown in Fig 8(a) and 

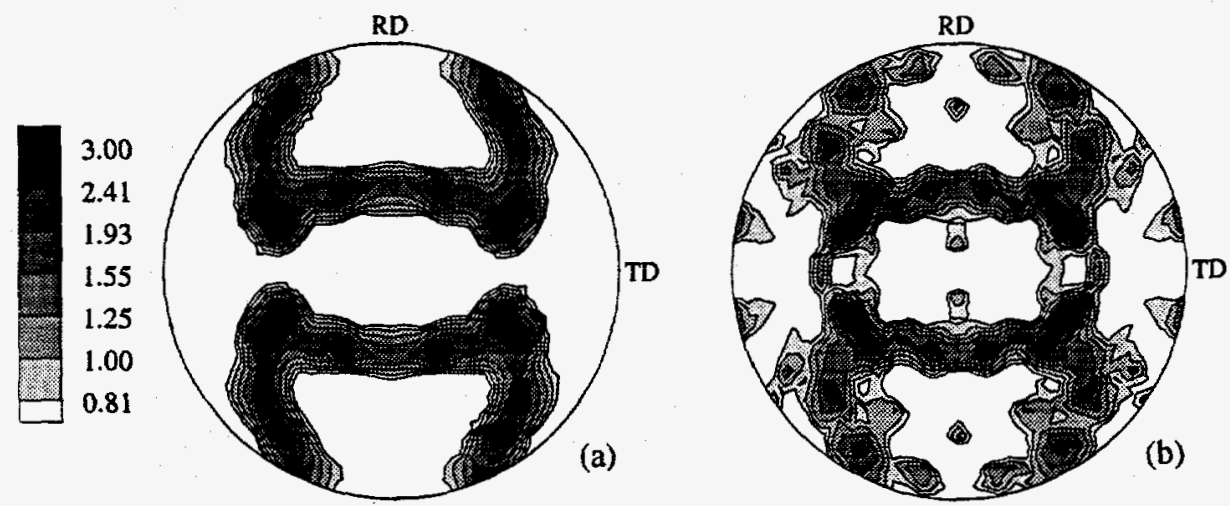

Figure 9: Deformation and recrystallization textures for the deformation condition 2 for the same section of the deformation volume shown in Fig. 8.

Fig 9(a). However, upon recrystallization, near-cube orientations (with a misorientation of about $13^{\circ}$ ) were obtained for the 18 slip system case as shown in Fig $9(\mathrm{~b})$. This result illustrates the fact that depending on the initial orientations in the microstructure, local variations in the stored energy and subgrain misorientations due to operation of different slip systems can lead to different recrystallization textures although the gross deformation textures are very similar.

\section{Conclusions}

The recrystallization of fcc polycrystals has been simulated with a technique that couples $\mathrm{FE}$ simulation of microstructural deformation with a novel $\mathrm{MC}$ technique that treats recrystallization as a heterogeneous subgrain growth process. The recrystallization simulation captures nucleation mechanisms based on heterogeneous growth of subgrains, and the migration of existing high angle boundaries, as well as the sweeping of the deformation substructure by the discontinuous growth of the nuclei. The observed recrystallization kinetics and microstructure, and their variation with extent of prior deformation are in agreement with general experimental observations. The model is able to capture the evolution of recrystallization textures and the dependence of deformation mode (operating slip systems) on the texture and kinetics. The model can also account for the nucleation and growth of cube and near-cube orientations during recrystallization. However, the full potential of this technique can only be realized with significant advances in computational power, that would permit the use of three-dimensional MC grid with much larger number of grains than in the current simulations.

\section{Acknowledgments}

This research was sponsored by the Office of Basic Energy Sciences, U.S. Department of Energy, under contract DE-AC05-96OR22464 with Lockheed Martin Energy Research Corporation. The research was supported in part by an appointment to the Oak Ridge National Laboratory Postdoctoral Research Associates Program administered jointly by the Oak Ridge National Laboratory and the Oak Ridge Institute for Science and Education. The authors acknowledge the use of the Intel Paragon XP/S 35 located in the Oak Ridge National Laboratory Center for Computational Sciences (CCS), funded by the Department of Energy's Office of Scientific Computing. 


\section{References}

1. D.J. Srolovitz, G.S. Grest and M.P. Anderson, "Computer Simulation of Recrystallization-I. Homogeneous Nucleation and Growth," Acta metall. 34 (1986) 1833-1845.

2. D.J. Srolovitz et al., "Computer Simulation of Recrystallization-II. Heterogeneous Nucleation and Growth," Acta metall. 36 (1988) 2115-2128.

3. A.D. Rollett et al., "Computer Simulation of Recrystallization-III. Influence of a Dispersion of Fine Particles," Acta metall. 40 (1992) 3475-3495.

4. A.D. Rollett et al., "Computer Simulation of Recrystallization in Non-uniformly Deformed Metals," Acta metall. 37 (1989) 627-639.

5. G. Sarma, B. Radhakrishnan and T. Zacharia, Proc. ReX' 96, The Third Int. Conf. on Recrystallization and Related Phenomena (Monterey, CA: Monterey Institute of Advanced Studies, 1997), 555-562.

6. B. Radhakrishnan, G. Sarma and T. Zacharia, "Modeling the Kinetics and Microstructural Evolution during Static Recrystallization," Acta mater. to appear.

7. R.D. Doherty et al., Proc. of the 16th Risø Int. Symp. on Materials Science: Microstructural and Crystallographic Aspects of Recrystallization (Roskilde, Denmark: Ris $\emptyset$ National Laboratory, 1995), 1-23.

8. F.J. Humphreys, "A Unified Theory of Recovery, Recrystallization and Grain Growth, Based on the Stability and Growth of Cellular Microstructures- I. The Basic Model," Acta. mater. 45 (1997) 4231-4240.

9. Cl. Maurice and J.H. Driver, "Hot Rolling Textures of f.c.c. Metals-Part I. Experi-" mental Results on Al Single and Polycrystals," Acta Mater., 45 (1997), 4627-4638.

10. B. Bacroix and J.J. Jonas, "The Influence of Non-Octahedral Slip on Texture Development in FCC Metals," Textures and Microstructures, 8 \& 9 (1988), 267-311.

11. G. Sarma, B. Radhakrishnan and T. Zacharia, "Polycrystal Simulations of Texture Evolution during Deformation Processing," these proceedings.

12. F.J. Humphreys and M. Hatherly, Recrystallization and Related Annealing Phenomena (Oxford, U.K: Elsevier Science Ltd., 1995), 17-18.

13. A.D. Rollett and E.A. Holm, Proc. ReX' 96, The Third Int. Conf. on Recrystallization and Related Phenomena (Monterey, CA: Monterey Institute of Advanced Studies, 1997), 31-41.

14. D.A. Hughes, "High Angle Boundaries Formed by Grain Subdivision Mechanisms," Acta. mater., 45 (1997), 3871-3886.

15. H.E. Vatne et al. "Modeling Recrystallization after Hot Deformation of Aluminium," Acta. mater., 44 (1996), 4463-4473. 